\title{
Microwave Resonant Cavities for Sensing Moisture and Mass of Single Seeds and Kernels
}

\author{
A. W. Kraszewski and S. O. Nelson \\ U.S. Dept. of Agriculture, Agricultural Research Service, Russell Research Center \\ P.O. Bo.1 5677, Athens, GA 306/3, USA
}

\begin{abstract}
Principles are presented for using microwave resonant cavities to sense properties of individual agricultural seeds and kernels. Results of research are presented illustrating the usefulness of the technique for size-independent measurements of seed and kernel moisture content and mass.
\end{abstract}

\section{INTRODUCTION}

When electrical properties of agricultural products are correlated with other characteristics of those products that are important, electric fields can often be used to sense those desired characteristics. Moisture content is an important characteristic in many agricultural products, because it affects their quality and storability. Electrical properties of materials are highly correlated with moisture content. Therefore, electrical measurements have been used very successfully for rapidly determining moisture content in such products as cereal grains and other seed crops.

Resonant cavities have been widely used for the measurement of microwave dielectric properties of certain materials [1]. It is the purpose of this paper to illustrate the manner in which microwave resonant cavities can be used for sensing such properties as moisture content and mass of individual agricultural products such as seeds and kernels of grain and peanuts.

\section{BASIC PRINCIPLES}

Resonant cavities excited at microwave frequencies have been used for permittivity measurements for a long time [2]. The measurement consists of determining the cavity parameters when empty and again when loaded with a sample of material. Two measurement parameters are then obtained: 1) the shift of resonant frequency $\Delta F=f_{0}-f_{s}$, where subscripts 0 and $s$ refer to the empty cavity and the cavity loaded with the sample, respectively; and 2) the change in the $Q$-factors

$$
\frac{1}{Q_{0}}-\frac{1}{Q_{0}}=\frac{1}{Q_{0}}\left(\frac{Q_{0}}{Q_{2}}-1\right)=\frac{\Delta T}{Q_{0}}
$$

U.S. Govemment work not protected by U.S. copyright where the transmission factor $\Delta T=\left(10^{k}-1\right)$, where $k=\left(S_{210}-S_{21 s}\right) / 20$, and $S_{21}$ is the voltage transmission coefficient of the cavity expressed in decibels.

It may be shown, that these two measurement parameters are directly related to the sample permittivity, shape, and volume by the following equations [2]:

$$
\begin{gathered}
\Delta F=2\left(\epsilon^{\prime}-1\right) K f_{0}\left(\frac{v_{o}}{v_{0}}\right) \\
\Delta T=4 \epsilon^{\prime \prime} K^{2} Q_{0}\left(\frac{v_{a}}{v_{0}}\right)
\end{gathered}
$$

where $K$ is a shape factor dependent upon sample shape, orientation and permittivity, and $v_{0}$ and $v_{0}$ are the volumes of the sample and the empty cavity, respectively.

The technique described here is based on the fact that dissipation (change in magnitude) and dispersion (change in resonant frequency) of the electromagnetic waves interacting with the sample in the cavity depend upon the permittivity of the sample. When moisture content changes, a change is reflected in wave parameters, $\Delta F$ and $\Delta T$. Because the relative permittivity of water differs significantly from that of most hygroscopic dielectric materials, like grain kernels, its effect can be easily separated from the effect of dry material. In general, it may be expressed in a functional form as $\Delta F=\Phi_{1}\left(m_{d}, m_{w}\right)$ and $\Delta T=\Phi_{2}\left(m_{d}, m_{w}\right)$ where $m_{d}$ and $m_{w}$ are the mass of dry material and mass of water, respectively. In practice, these relationships are determined empirically. In most practical cases, the two equations can be solved simultaneously to express the mass of water and the mass of dry substance in terms of two measured parameters in the form: $m_{d}=\Psi_{1}(\Delta F$, $\Delta T)$ and $m_{w}=\Psi_{2}(\Delta F, \Delta T)$. Substituting the analytical expressions corresponding to $\Psi_{1}$ and $\Psi_{2}$ into the definition of moisture content, $M$ in percent, wet basis, one obtains the following relationship:

$$
\begin{gathered}
M=\frac{m_{w}}{m_{d}+m_{w}} \times 100 \\
=\frac{\Psi_{2}(\Delta F, \Delta T)}{\Psi_{1}(\Delta F, \Delta T)+\Psi_{2}(\Delta F, \Delta T)} \times 100
\end{gathered}
$$


which contains only the wave parameters determined experimentally and is independent of the size of the sample.

It has been shown [3] that use of equations (2), (3), and (4) permits a determination of moisture content for nearly spherical seeds (soybeans, Glycine $\max$ L.) independent of size variation among the seeds. For irregularly shaped seeds or kernels such as corn, Zea mays. L., this ratio is also useful, but two measurements of $\Delta F$ and $\Delta T$, with kernel orientations separated by a $90^{\circ}$ rotation with respect to the $E$-field vector, are necessary, and the averaged values of these variables can be used to provide a moisture determination independent of size and shape [4]. For regularly shaped kernels, such as peanuts, Arachis hypogaea L., single measurements of $\Delta F$ and $\Delta T$ can be used to obtain both moisture content and mass of the kernels simultaneously [5].

\section{MEASUREMENTS}

All measurements were carried out with resonant cavities consisting : of standard rectangular waveguide sections coupled with external waveguides through small circular holes in shortcircuiting brass plates. Each cavity was located between two waveguide-to-coaxial-line transitions that allowed it to be connected to an automatic network analyzer. The network analyzer was calibrated in the transmission mode. When used with 801 discrete frequency points within a range of $16 \mathrm{MHz}$, it permitted the determination of the resonant frequency within increments of $20 \mathrm{kHz}$.

Soybean seeds and corn kernels were inserted into the cavities through small ports in the wide wall of the waveguide at the center of the cavity in such a way that cavity configuration was maintained constant. Corn kernels were positioned at the center of the S-band cavity by a $0.33-\mathrm{mm}$ nylon line which passed through the kernels and was held by circular fittings on each side of the cavity exterior. These fittings were provided with dial markings for accurate rotation of the kernel to obtain measurements separated by $90^{\circ}$.

The S-band cavity used for peanut kernel measurements was identical to the one used for corn kernels, except that it was fitted with a vertical polystyrene tube passing through the center of the cavity through which the kernels could be introduced into the cavity.

The initial moisture content of the soybean lot was about 8 percent, and water was added to provide higher moisture levels. Both the corn and the peanuts were measured soon after harvest, so the initial measurements were taken at natural moisture levels, and the kernels were then dried under ambient conditions (about $24^{\circ} \mathrm{C}$ and 30 percent relative humidity) between measurements. The soybeans were also permitted to dry between measurements, and with all three, the individual seeds or kernels were sealed individually in small glass vials for moisture distribution to equilibrate for at least 24 hours between drying intervals and the next measurements.

When ready for the cavity measurements, the resonant frequency and transmission factor were measured for the empty cavity. Then samples were placed into the cavities one at a time and the corresponding values of the same two parameters were determined with the sample in the cavity. The frequency shift $\Delta F$ and the change in the transmission factor $\Delta T$ were then computed.

At the completion of the measurement series, the individual seeds and kernels were dried under standard specified conditions in a forced-air oven, and dry weights were determined for calculation of moisture contents at each level used for the cavity measurements.

\section{EXPERIMENTAL RESULTS}

\section{Size-Independent Determination of Moisture Content in Objects of Uniform Shape}

The determination of moisture content in single soybean seeds is an example of applying equations (2) and (3) to objects of nearly spherical shape. The permittivity (dielectric properties) of the seeds is constant for a given moisture content. The cavity parameters, measured simultaneously for each seed positioned at the center of the cavity, $\Delta F$ and $\Delta T$, are both proportional to the volume of the seed and its moisture content, and both depend upon the shape of the seed. It has been proven [3] that the ratio of these two parameters is independent of size (volume) of the seed, and slight variations in seed shape do not significantly affect the dependence of this ratio upon moisture content. It may be observed from the equation

$$
X=\frac{\Delta F}{\Delta T}=\frac{\epsilon^{\prime}-1}{\epsilon^{\prime \prime}} \frac{1}{K} \frac{f_{0}}{2 Q_{0}}
$$

that the ratio $X$ is a defined function of the sample permittivity for any given cavity.

The dependence of the variable $X$ on seed moisture content, determined by the standard oven method, was described by a simple empirical equation which fit a set of 108 experimental data points obtained with the cavity operating at $6.0 \mathrm{GHz}$ in the $\mathrm{TE}_{10}$ mode [3]. This calibration equation had the form.

$$
1 / X=0.00233 M-0.0021
$$


with correlation coefficient $r=0.9912$. Simple conversion of the above equation gave a calibration equation for moisture content determination in the form

$$
M=\frac{430}{X}+0.9
$$

Verification of this calibration equation for another set of soybean seeds indicated the mean value of differences between oven moisture content and moisture content calculated from equation (7) as $0.15 \%$ moisture. The standard deviation of the differences, standard error of performance (SEP), was $0.51 \%$ moisture. Although these results were satisfactory for soybeans of quite uniform shape, a different approach had to be taken for moisture content determination in single corn kernels, which have irregular shapes.

Size-Independent Moisture Content Determination in Arbitrarily Shaped Kernels

Experiments with corn kernels illustrate the use of equations (2) and (3) for moisture content determination on objects of variable size and shape. Among 108 kernels tested in an S-band resonant rectangular cavity, the highest-to-lowest weight ratio was 2.1. During these measurements, it was observed that when a corn kernel of arbitrary shape is rotated about the $x$-axis in the cavity, both $\Delta F$ and $\Delta T$ follow $\cos ^{2} \theta$ behavior, where $\theta$ is the angle of rotation [4]. Because the sum of $\cos ^{2} \theta$ for two arbitrary angles differing by $90^{\circ}$ is constant, the shape effect in equation (5) can be nearly eliminated by taking two independent measurements of $\Delta F$ and $\Delta T$ with the kernel oriented in two positions differing by $90^{\circ}$. Averaged values of these variables are then used for calculations.

Measurements of this type were taken at $3.2 \mathrm{GHz}$ on 108 kernels of yellow-dent field corn ranging in moisture content from 9 to $18 \%[6]$. The averaged shift of resonant frequency $\Delta F$ and the averaged value of transmission factor $\Delta T$ as a function of mass of water $m_{w}$ and mass of dry kernel $m_{d}$ were expressed by the two following linear equations:

$$
\begin{gathered}
\Delta F=-0.018+0.05777 m_{w}+0.00755 m_{d} \\
r=0.9849 \\
\Delta T=-0.042+0.01575 m_{w}+0.000016 m_{d} \\
r=0.9682
\end{gathered}
$$

where $m_{w}$ and $m_{d}$ are expressed in $m g$. Solving the two equations simultaneously permitted the mass of water and the mass of dry kernel to be expressed in terms of measured values. Then in turn they were used to express the kernel moisture content $M$, in percent, wet basis, as follows:

$$
M=\frac{100 m_{w}}{m_{w}+m_{d}}=\frac{47.9+17.45 Y-0.101 X}{X-3.18-\mathrm{Y}}(8)
$$

where $X=\Delta F / \Delta T$ and $Y=0.1157 / \Delta T$. This expression provides for the determination of kernel moisture content without regard to the kernel dimensions or shape. The verification of the calibration equation (8) was performed on another 80 kernels of moisture content ranging from 9 to $18 \%$. The mean value of the differences between oven moisture content and moisture content calculated from equation (8) was $0.25 \%$ moisture, and the SEP was $0.61 \%$ moisture.

Determination of Moisture Content and Mass of Uniformly Shaped Objectss

Peanut kernels have regular prolate ellipsoidal shape with a major-to-minor-axis ratio of about 2 . Forty-five peanut kernels of moisture contents from 10 to $14 \%$ and of both medium and jumbo sizes were randomly selected for the experiment. The weight of wet kernels ranged from 400 to $900 \mathrm{mg}$. With the cavity operating at $3.96 \mathrm{GHz}$ in the $\mathrm{TE}_{107}$ mode, 168 measured points were obtained on the 45 kernels. Linear relationships were obtained between both $\Delta F$ and $\Delta T$ as functions of the mass of water $m_{w}$ and dry matter mass $m_{d^{*}}$. Two linear equations fit the experinnental results with a high statistical significance:

$$
\begin{gathered}
\Delta F=-0.308+0.133726 m_{w}+0.00103 m_{d} \\
r=0.9880 \\
\Delta T=-0.062+0.017174 m_{w}-0.0004 m_{d} \\
r=0.9891
\end{gathered}
$$

These two equations can be solved simultaneously for $m_{w}$ and $m_{d}$ to provide the calibration equations for the wet-kernel mass, $m_{m}$, and kernel moisture content in the form

$$
\begin{gathered}
m_{m}=m_{w}+m_{d} \\
=73.155 \Delta F-51.0 .358 \Delta T-13.372 \\
M=\frac{100 m_{w}}{m_{w}+m_{d}} \\
=\frac{0.17 \Delta F+4.377 \Delta T+0.452}{0.07315 \Delta F-0.5104 \Delta T-0.0134}
\end{gathered}
$$

Equations (9) and (10) were then used for calculation of the wet mass of individual kernels and their moisture content, respectively, from the resonant cavity measurements. The calculated values were compared with those obtained by standard oven methods. The results are shown in Figs. 1 and 2. The predicted values of peanut mass presented in Fig. 1 agreed with those obtained by 
weighing with an accuracy of $\pm 18 \mathrm{mg}$ in the whole range of moisture content from 4 to $14 \%$. The predicted values of peanut moisture content shown in Fig. 2 agreed well with those determined by the standard method, and the standard error of calibration (SEC) was $0.37 \%$ moisture content. It should be noted that the uncertainty in the standard oven moisture determinations is generally $\pm 0.2 \%$ moisture content. Thus, by measuring two parameters of the resonant cavity loaded with a peanut kernel, its size and moisture content might be determined immediately and without any contact between the kernel and the measuring instrument.

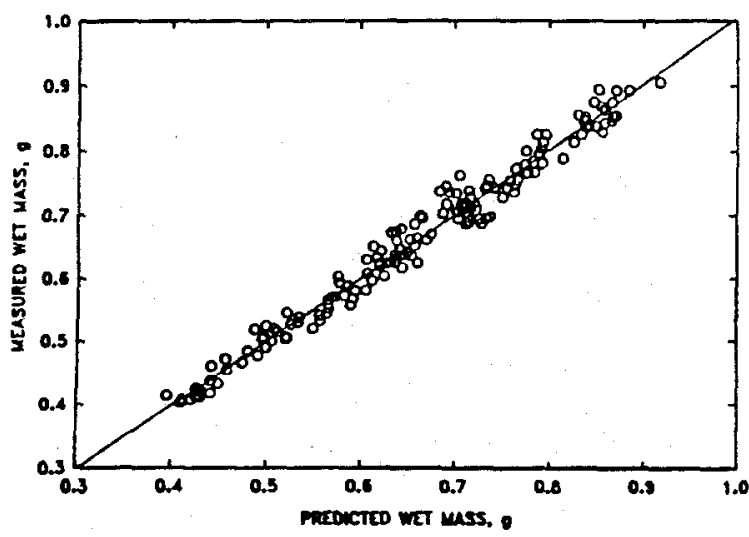

Figure 1. Mass of wet peanut kernels predicted by equation (9) versus kernel mass determined by precision weighing.

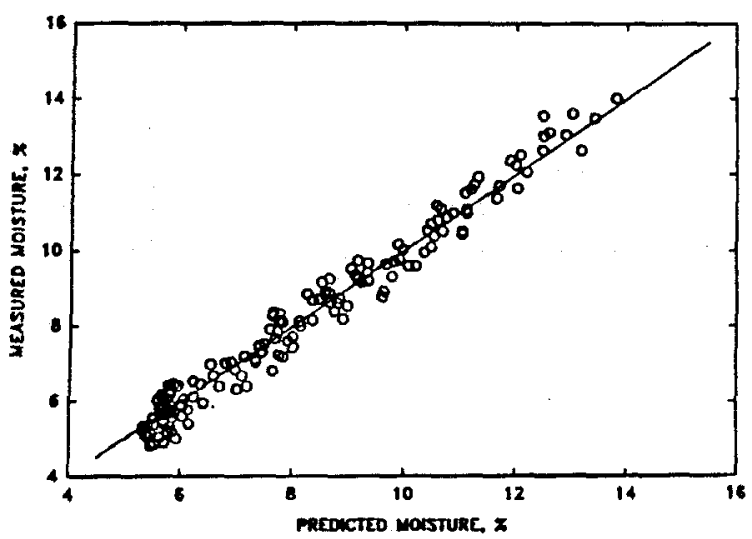

Figure 2. Moisture content of mixed medium and jumbo size peanut kernels predicted by equation (10) versus moisture content determined by standard oven methods.

\section{CONCLUSIONS}

Microwave resonant cavities can be used to sense the moisture content (m.c.) of spherically shaped seeds such as soybeans by simultaneous measurement of the frequency shift $\Delta F$ and the change in the cavity transmission factor $\Delta T$. The ratio $X=\Delta F / \Delta T$ provides an accurate (SEP =
$0.5 \%$ m.c.) moisture determination independent of seed size.

Moisture content of objects of varying shape and size, such as kernels of corn, can also be determined accurately ( $\mathrm{SEP}=0.6 \%$ m.c.), but $\Delta F$ and $\Delta T$ measurements at two orientations differing by $90^{\circ}$ rotation are required. The resulting moisture determination, however, is independent of kernel shape and size.

Both moisture content and mass of objects of uniform shape, such as peanut kernels, can be determined by a single measurement of $\Delta F$ and $\Delta T$ when the kernels are presented in uniform orientation with respect to the electric field vector in the cavity.

Thus, resonant cavity measurements offer a method for sensing moisture content of individual seeds and kernels of grain and peanuts without the need for contact between the objects being measured and the sensing system.

\section{References}

[1] Bussey, H. E., "Measurement of RF properties of materials - A survey," Proc. IEEE vol. 55, no. 6, pp. 1046-1053, 1967.

[2] Altschuler, H., "Dielectric constant," Chapter IX, pp. 495-546, in Handbook of Microwave Measurements, M. Sucher and J. Fox, eds., vol. 2, pp. 530-536, 1963. New York: Polytechnic Press, Brooklyn.

[3] Kraszewski, A. W., T.-S. You and S. O. Nelson, "Microwave resonator technique for moisture content determination in single soybean seeds," IEEE Trans. Instrum. Meas., vol. 38, no. 1, pp. 79-84, 1989.

[4] Kraszewski, A. W., S. O. Nelson and T. -S. You, "Use of a microwave cavity for sensing dielectric properties of arbitrarily shaped biological objects," IEEE Trans. Microwave Theory Techn., vol. 38 , no. 7 , pp. $858-863,1990$.

[5] Kraszewski, A. W., and S. O. Nelson, "Microwave resonator as a dielectric object sorting device: advantages and limitations," 21st European Microwave Conf. Proc., vol. 1, pp. 435-440, Stuttgart, Germany, Sept. 9-12, 1991.

[6] Kraszewski, A. W., S. O. Nelson, and T.-S. You, "Moisture content determination in single corn kernels by microwave resonator techniques," J. Agric. Eng. Res., vol. 48, pp. 77-87, 1991. 